Advanced X-Ray scattering methods for the study of structure and its evolution in soft materials with fiber symmetry

This article has been downloaded from IOPscience. Please scroll down to see the full text article.

2010 IOP Conf. Ser.: Mater. Sci. Eng. 14012003

(http://iopscience.iop.org/1757-899X/14/1/012003)

View the table of contents for this issue, or go to the journal homepage for more

Download details:

IP Address: 95.118.126.165

The article was downloaded on 26/02/2011 at 19:20

Please note that terms and conditions apply. 


\title{
Advanced X-Ray scattering methods for the study of structure and its evolution in soft materials with fiber symmetry
}

\author{
Norbert Stribeck \\ University of Hamburg, Dept. of Chemistry, Inst. of Technical and Macromolecular Chemistry, \\ Bundesstr. 45, 20146 Hamburg, Germany \\ E-mail: Norbert.Stribeck@desy.de
}

\begin{abstract}
Three recently developed evaluation methods for the automated quantitative analysis of Xray scattering data (small-angle (SAXS) and wide-angle (WAXS)) are presented. They are applicable to extensive series of 2D patterns that are recorded in studies of polymer materials with uniaxial symmetry. The experiments comprise time-resolved studies (melting, crystallization, mechanical properties and fatigue) as well as microbeam-scanning for the study of nanostructure gradients. The methods appear suitable to manage the data flood from modern synchrotron radiation setups aiming at the extraction of quantitative information on the structure evolution inside the material.

In microbeam-scanning experiments the recorded scattering patterns are smeared. It is proposed to reconstruct desmeared scattering patterns by an X-ray scattering fiber-computer-tomography (XS-FCT). Reconstruction aberrations yield additional structure information. The true structure variation along the fiber radius is established. Compared to general tomography the experiment is faster by a factor of 100 , and reconstruction is faster by a factor of $10^{4}$. In WAXS fiber patterns should first be mapped into reciprocal space before analysis. After having corrected an erroneous tilt-angle equation, automatic tilt-angle tracking and mapping becomes possible. If polymers fail at low strain, the determination of strain and of structural parameters from the scattering patterns require very high accuracy because the observed variations are subtle. Suitable methods are presented both for the high-precision determination of the macroscopic strain, and for the determination of subtle variations of structure parameters.
\end{abstract}

\section{Introduction}

Intense, reliable X-ray sources have become generally available at synchrotron facilities. At such sources less than a second is sufficient to expose a low-noise two-dimensional (2D) scattering pattern of an oriented polymer sample. Low noise is required for a quantitative analysis, and anisotropy increases the structure information contained in the pattern. Combined with the most advanced detectors, cycle times of less than a second can be realized. The results of respective experiments offer the potential to strengthen the understanding of structure evolution mechanisms in polymers. Moreover, the new instruments provide very narrow X-ray beams with diameters of less than $1 \mu \mathrm{m}$ ("microbeams") that permit to study the variation of structure inside the material with the spatial resolution of the beam diameter. Anticipating the expected increase of both quality and data quantity, new methods are required for the quantitative analysis of extensive series of scattering patterns.

Because the scattering of isotropic materials exhibits a rather low information content, their analysis requires substantial model assumptions. This problem is relieved, if oriented materials are studied. Among these materials systems with cylindrical symmetry ("fiber symmetry") play a particularly 
important role. The reason is that many interesting materials show this kind of symmetry. This class of materials contains synthetic and natural polymer fibers, rods, and extruded parts. For such of materials almost the complete scattering information contained in 3D reciprocal space can be recorded in a single 2D detector frame. Only the size of the detector and the curvature of the Ewald sphere limit $[1,2]$ the accessible information, the latter causing information loss at wide angles near the meridian (i.e. in the direction of the fiber axis). Thus, unique time-resolved scattering experiments can be carried out with polymer fibers in order to monitor structure evolution, because the complete scattering information can be recorded in a single snapshot. In similar manner it becomes particularly simple to unravel the smearing of the structure gradient in polymer fibers, if 2D scattering patterns from a microbeam scan across the fiber have been measured.

The anisotropic scattering of materials with fiber symmetry can be recorded almost completely on a 2D detector. This kind of completeness renders application of scattering theory particularly promising. Based on respective considerations, quantitative data analysis methods can be devised that return wellfounded results on structure variation as a function of time and space, respectively. In the present work 3 methods are presented that have recently been developed in our group in order to evaluate extensive sets of $2 \mathrm{D}$ scattering patterns from polymer materials with fiber symmetry.

\section{Structure gradients in polymer fibers}

Scientific problem. The structure inside a polymer part may be inhomogeneous. With fibers and extruded strands a core-shell structure is frequent. If such a part is irradiated by an X-ray beam, the recorded pattern is an integral superposition of all the SAXS patterns emerging from the sequence of volume elements (voxels) along the beam path. Such a superposition yields smeared structure information, but the fact that the superposition is a mathematical projection is a good starting point for resolving the problem. The required experimental technique is microbeam scanning [3-5], which has been developed long ago. Here the lateral spatial resolution is only limited by the diameter of the beam. Nevertheless, along the beam the structure information remains smeared.

The solution of the problem is tomographic reconstruction. Its implementation is aggravated from the fact that scattering patterns are multidimensional arrays of numbers instead of a single number, as is the case in classical tomography. Thus, tomographic reconstruction of scattering data is difficult. An exception is the case in which uniaxially oriented parts are studied. In this case an approximate reconstruction of the scattering pattern is possible that has exclusively emerged from any chosen voxel located in the irradiated plane perpendicular to the "fiber" axis [6]. Unfortunately, this method is only of academic value. The assumptions on the structure to be studied under which the reconstruction is satisfactory are quite severe. Moreover, the exposure time for the recording of one set of low-noise patterns is still in the order of days and the reconstruction takes a week of computing time, even if the recorded patterns are binned to $128 \times 128$ pixels.

Solution: X-ray scattering fiber computer tomography. A more practical tomographic method can be devised, if the part to be studied both exhibits macroscopic fiber symmetry, and the structure only varies as a function of the distance from its central axis. By means of this method fibers, pipes, and extruded strands can be investigated. We call it "X-ray scattering fiber computer tomography" (XSF-CT). A complete set of projected scattering patterns is collected in a single microbeam sweep across the fiber, because the set of projections does not change as the sample is rotated about its axis. Compared with full tomography, the recording time is shorter by a factor of at least 100, and the reconstruction time is shorter than the recording time. Moreover, the assumption of a structure with "local fiber symmetry" (LFS cf. Fig. 1) is sufficient to guarantee perfect reconstruction, and deviations of the real structure from this assumption lead to well-defined $[6,7]$ reconstruction aberrations from which additional information on the structure itself is obtained. Last but not least, this technique is fast enough to sweep the microbeam across the fiber while the specimen is subjected to thermal or mechanical load. 


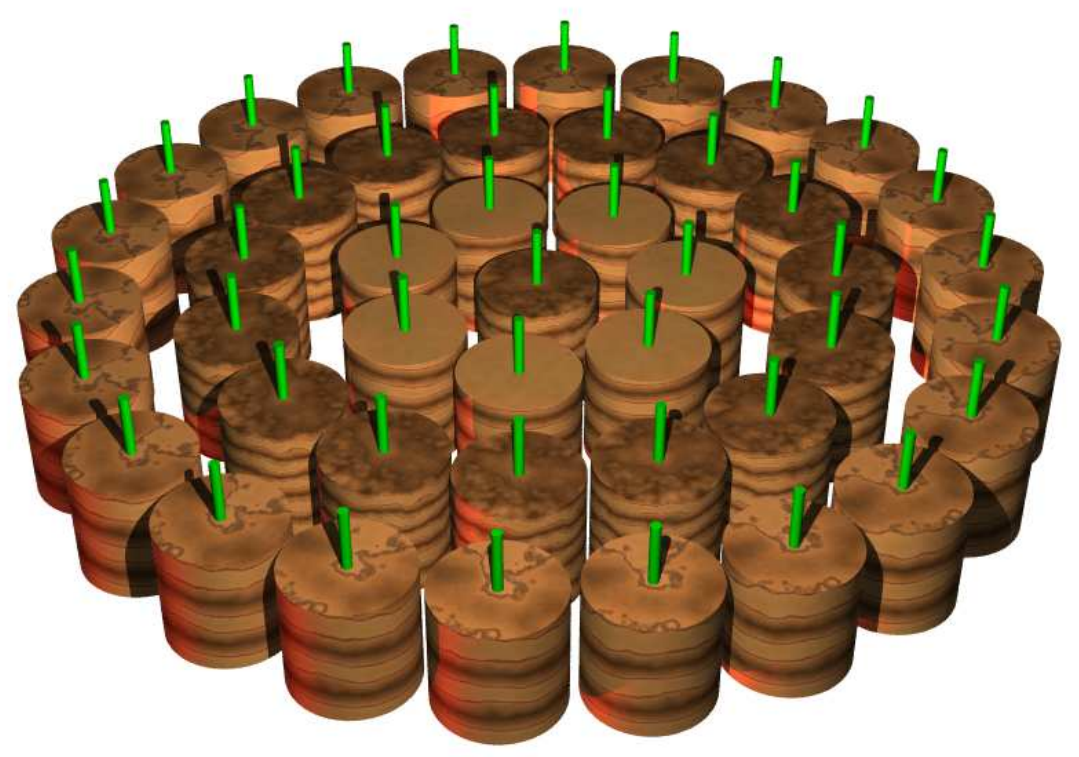

Figure 1. noneSketch of local fiber symmetry inside the cross-section of the fiber. Each voxel has its own fiber axis (green axes). The cylindrical shape of the voxels is a simplification

In a general tomographic X-ray experiment [8], a voluminous sample is scanned by a thin X-ray beam. As a function both of the position $x$ of the scanning beam on the sample, and of the sample rotation angle $\phi$, projections (notation: \{\}$)$ of the absorption $\{A\}(x, \phi)$ or even of complete scattering patterns $\{I\}(\mathbf{s}, x, \phi)$ are measured, in order to analyze the structure variation in the plane of the sample that is scanned by the X-ray beam $[6,9,10]$. Here $\mathbf{s}$ is the scattering vector with $|\mathbf{s}|=s=(2 / \lambda) \sin \theta$, the X-ray wavelength $\lambda$ and the scattering angle $2 \theta$. Tomographic image reconstruction $[8,11,12]$ returns either the spatial variation of the absorption in the plane, or of the scattering emanating from the individual voxels in the plane. The aim is to reconstruct a clear image of the inner structure.

Assuming cylindrical symmetry, the fundamental geometry is sketched in Fig. 2a. The information in the measured pattern $\{I\}(\mathbf{s}, x)$ does not represent the sought information $I(\mathbf{s}, x)$ originating from the small square (voxel) around the position $x$. Instead, to a first approximation it is represented by the projection integral

$$
\{I\}(\mathbf{s}, x)=2 \int_{x}^{\infty} I\left(\mathbf{s}, \sqrt{x^{2}+y^{2}}\right) d y .
$$

The sought information in image space $\left(I\left(\rho_{f}\right)\right)$ along the radius $\rho_{f}\left(\rho_{f}^{2}=x^{2}+y^{2}\right)$ of the fiber has to be reconstructed from the information in projection space $(\{I\}(x))$.

Eq. (1) is the definition of the Abel transform [12]. In X-ray scattering Eq. (1) is established textbook knowledge [13-19]. There it describes the slit smearing. Even the inverse Abel transform [20]

$$
I\left(\mathbf{s}, \rho_{f}\right)=-\frac{1}{\pi} \int_{0}^{\infty} \frac{d\{I\}(\mathbf{s}, x)}{d x} \frac{d y}{x}
$$

is found in scattering textbooks since Guinier [21] and DuMond [22]. Similar to the filtered backprojection algorithm of the general tomography, low-noise reconstruction algorithmsnone [23-25] for the tomography of materials with cylindrical symmetry are readily available. The idea of the corresponding 1D tomographic reconstruction is sketched in Fig. 2b. 

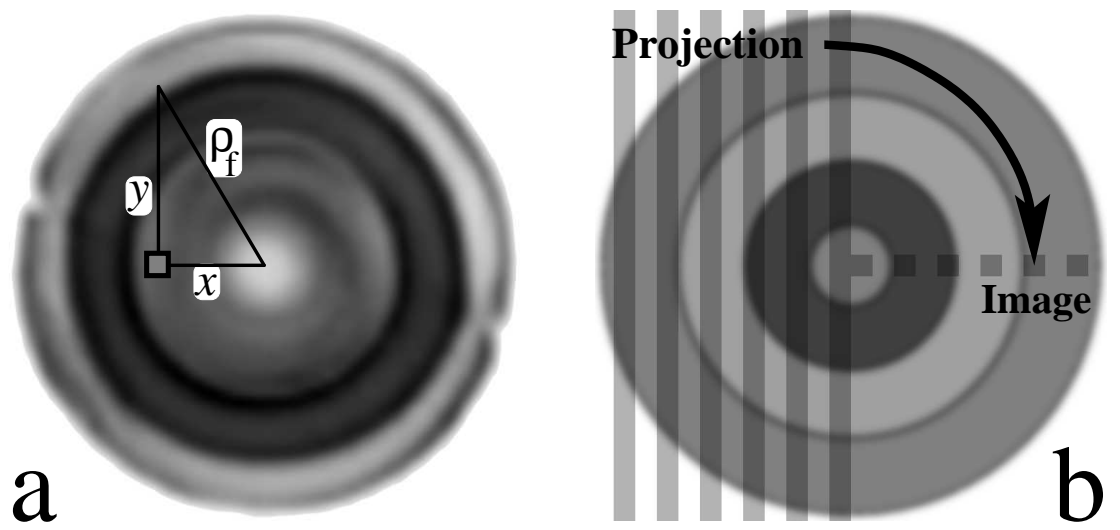

Figure 2. none(a) Imaged PE fiber cross-section from SAXS absorption tomography and the geometrical relations of the microbeam experiment for the X-ray beam penetrating the sample at an offset $x$ from its center. Fiber symmetry is a good approximation for the structure $\rho\left(\rho_{f}\right)$. The beam probes a superposition of all structures along its path, $y$. (b) One-dimensional tomographic reconstruction turns the measured series of projected scattering patterns that carry the structure information accumulated by the beams (vertical bars) into the image patterns from voxels (quadratic boxes) residing on the fiber radius

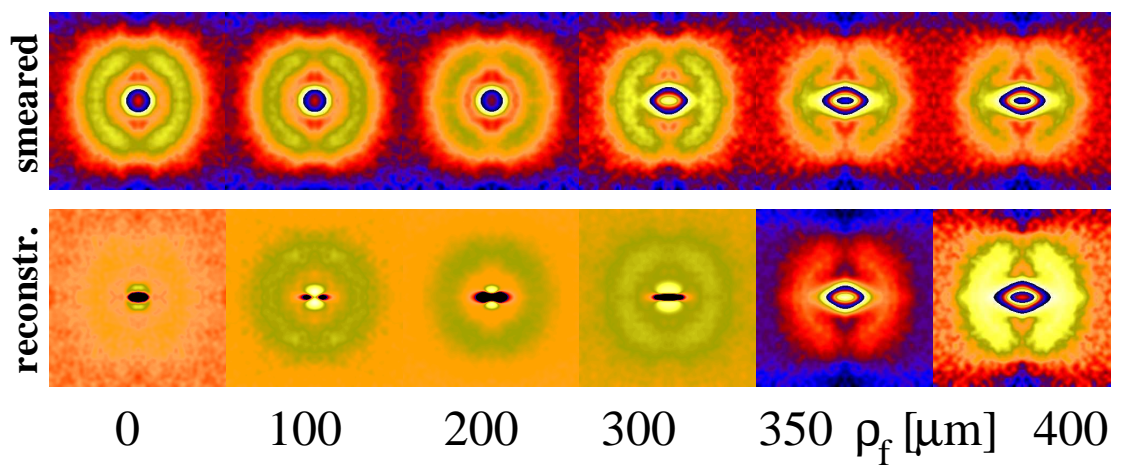

Figure 3. Microbeam-scan SAXS patterns of an extruded PEBA strand. The fiber direction $s_{3}$ is vertical, and $s_{12}$ is the equatorial direction. The patterns display the range $-0.2 \mathrm{~nm}^{-1} \leq s_{12}, s_{3} \leq 0.2 \mathrm{~nm}^{-1}$ in uniform logarithmic scale $(s=(2 / \lambda) \sin \theta)$. Top row: Measured patterns. Bottom row: XSF-CT reconstructed patterns as a function of the distance $\rho_{f}$ from the axis of the strand

Application. In an application-oriented feasibility study [7] composites based on poly(ether)-blockamide (PEBA) are studied. The results from a direct analysis of the smeared measured patterns are compared to results obtained after tomographic reconstruction. Ideas for advanced practical applications of the XSF-CT method are discussed. Figure 3none shows an example. All measured patterns (top row) show a short equatorial streak and strong, arc-shaped reflections at the equator with a long period of $10 \mathrm{~nm}$. Thus, the hard domains of the PEBA appear arranged preferentially transverse to the fiber direction. The tomographic analysis returns a more detailed view. Up to $\rho_{f}=300 \mu \mathrm{m}$ there is little orientation. The isotropic long period is $10 \mathrm{~nm}$. Let us address the obvious violations of LFS in this fiber. In the core of the strand negative-intensity equatorial streaks indicate over-reconstruction. Thus, at least a fraction of the equatorial streak observed in the shell zone must be attributed [7] to shingle-shaped voids in radial orientation that violate LFS. The central voxel $\left(\rho_{f}=0\right)$ does not fit into the series. The reason is, that another type of LFS violation ("tangential grain" [7]) leads to a reconstruction aberration that is always accumulated in the central voxel of the reconstructed SAXS. 


\section{Mapping of WAXS patterns}

Scientific problem. Wide-angle X-ray diffraction (WAXD) patterns of fibers are mapped [26] from the detector plane into reciprocal space before they are analyzed quantitatively. A manual refinement of each pattern appears inacceptable in materials science, where hundreds of patterns per hour are accumulated to monitor structure evolution. A fast and automated procedure is required. Because the fiber tilt may vary during the experiment, the algorithm must be able to track and to compensate such variation.

Solution: Error in geometric equation corrected. It may be worth mentioning that fiber mapping is a problem of pure mathematics. There is a fundamental philosophical difference between mathematical problems and problems from the applied sciences. In the applied sciences it is good practice to search for approximations and to refine them. Concerning a mathematical theorem, the related equation is either correct or wrong. In particular, if a published solution to a geometrical problem is addressed an "approximation" $[26,28]$ later, it should be clear that the respective problem is still waiting for a rigorous solution.

After revisiting the theoretical treatment of the fiber mapping it has been demonstrated [27] that there is no principal reason to refine an approximate center of the fiber pattern iteratively. Moreover, instead of an approximation [26,28] of the tilt angle $\beta$ of the fiber an exact equation [27] can be employed. In a methodical paper [29] an algorithm is presented by which the mapping can be performed automatically. The method is unsuitable for diffuse scattering patterns. If inaccuracies of 2 pixels can be tolerated, refinement can be avoided and the pattern is automatically mapped into reciprocal space in real time.

The procedure. For each series of diffraction patterns from a time-resolved experiment, some mapping parameters must once be determined interactively. Our procedure wf_premap [30] assumes that the studied material exhibits a sharp reflection that is located neither on the equator nor on the meridian. In the example [31] we investigate polypropylene and select its (131) reflection. With the crystallographic caxis parallel to the meridian, the reflection is characterized by the parameters [32] $d_{h k \ell}=d_{131}=0.406 \mathrm{~nm}$ and by $c / \ell=0.6504 \mathrm{~nm}$ that defines the position of the reflection ring on the Polanyi sphere $[1,2,27]$. The wavelength of $\mathrm{X}$-radiation is $\lambda=0.15 \mathrm{~nm}$. With these parameters the first pre-mapping run of the series is started [33] by

wave $>a b=w f \_p r e m a p(s s, a, 0.15,0.406,0.6504)$

The procedure requires a diffraction pattern as input (a). It generates output both in a "save-set" (ss), and in a background-corrected and mapped diffraction pattern $(\mathrm{ab})$. If the save-set is still undefined, the procedure enters interactive mode and the user is presented the pattern as shown in Fig. 4a.

Obviously, in Fig. 4a the meridian is not vertical and the fiber is tilted. Now the user draws the reflection circle through the maxima of the reflection spots of the (131)-reflection. Thereafter he transforms the circle into a belt that is wide enough to contain the maxima of the spots (Fig. 4a). Now the user specifies 4 disjoint clips (regions of interest on the reflection circle). This is done by punching out circular regions from the belt (the bottom right one is shown in Fig. 4a). The clips are the intersections of the belt (white double circle) and the smaller circles. The program determines the positions of the maxima in the clips. From these 4 maxima-positions the best reflection circle is determined by regression. The orientation of the meridian is computed. Based on this information the diffraction pattern is centered and aligned. Finally, the program computes the tilt angle $\beta$ of the fiber from the upper and lower orientation angles [27] of the spots, $\delta$ and $\delta^{\prime}$, respectively, using the exact geometric equation [27]

$$
\begin{aligned}
\tan \beta & =\frac{\sqrt{4-\lambda^{2} s_{r}^{2}}}{2 \lambda s_{r}}\left(\cos \delta^{\prime}-\cos \delta\right) \\
& =\frac{2 \cos \theta_{r}}{\sin \theta_{r}}\left(\cos \delta^{\prime}-\cos \delta\right)
\end{aligned}
$$



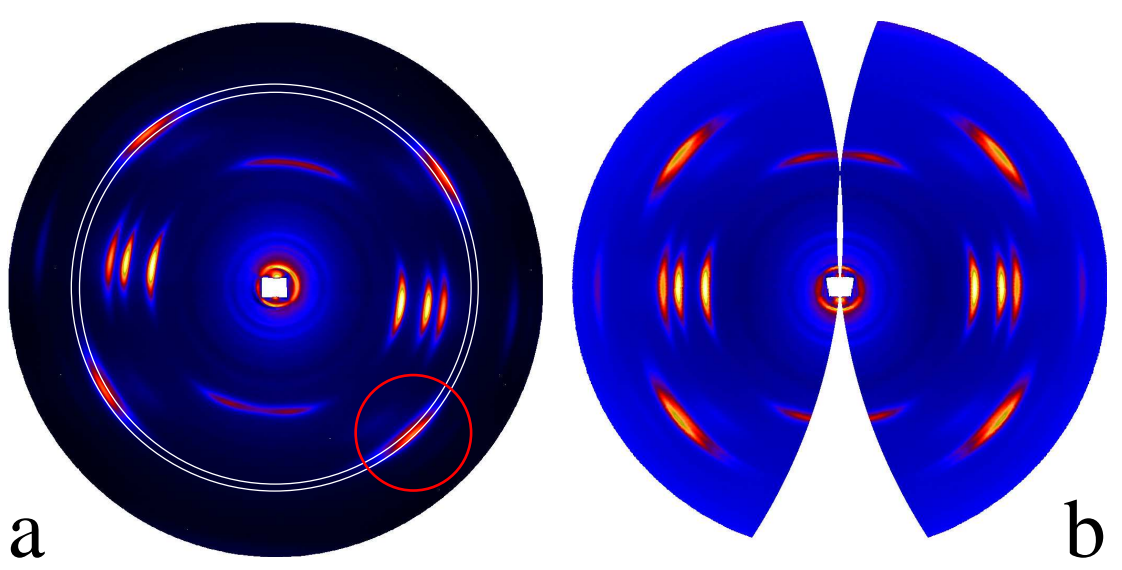

Figure 4. (a) Interactive mode of wf_premap: Draw the reflection circle through the centers of the reflection spots of an internal standard reflection (here: polypropylene (131) reflection). Widen the circle into a belt that contains the maxima of the reflection spots. Finally input 4 circles (one indicated in red).

(b) The procedure wf_map has mapped the fiber diffraction pattern into reciprocal space

Here $s_{r}=1 / d_{131}$ is the radius of the Polanyi sphere of the reference reflection and $\theta_{r}$ is the Bragg-angle of the considered reflection. The wrong equation published by Franklin and Gosling [28] is obtained after replacing $\cos \theta_{r}=1$ for all $\theta_{r}$. Finally, the save-set SS is filled with the data required for an automatic processing of the complete series of diffraction patterns recorded in a time-resolved X-ray diffraction experiment of materials with fiber symmetry. If the procedure wf_premap is called with a well-defined save-set, the interactive part is skipped, and the geometry of the 4 clips is taken from the save-set. From the peak positions in the clips the actual fiber tilt angle $\beta$ is computed. Finally, the mapping into reciprocal space is accomplished by the routine [30] wf_map. The function call

wave $>$ rec=wf_map $(\mathrm{ss}, \mathrm{ab})$

maps the diffraction pattern ab into reciprocal space using the save-set ss. The result is rec. A detailed description is in the original paper [29]. Figure $4 \mathrm{~b}$ shows a typical result obtained by automatic direct mapping. Because the result pattern is in reciprocal space, it should exhibit symmetry in 4 quadrants. Thus, the quality of the mapping can be assessed by comparison to a 4-quadrant average of the pattern.

Application. In a WAXD study [31], uniaxially oriented polypropylene (PP) is molten and crystallized isothermally from the oriented, quiescent melt. The material bends during temperature treatment. Figure 5 shows the fiber tilt angle $\beta(t, T)$ from one of the experiments. The smooth tracking curve demonstrates the reliability of the tilt-angle determination.

After the mapping the intensity distribution is known almost everywhere in reciprocal space except for a wedge region near the meridian (cf. Fig. 4b). Thus, reflection intensities can readily be integrated in reciprocal space. In reciprocal space the relation between scattering and structure is clear from scattering theory [19,31], and structural data can be computed [31], e.g., from total intensities (crystallinity) and integral breadths (crystallite sizes).

Figure 6 shows the evolution of the weight crystallinities of the two differently oriented sets of crystallites ( $c$-set and $a^{*}$-set) at 3 different crystallization temperatures. Latency periods between the quench and the start of the crystallization, as well as crystallization velocities of the two kind of crystallites are extracted from these data. Finally, conclusions concerning the crystallization mechanisms can be drawn [31]. 


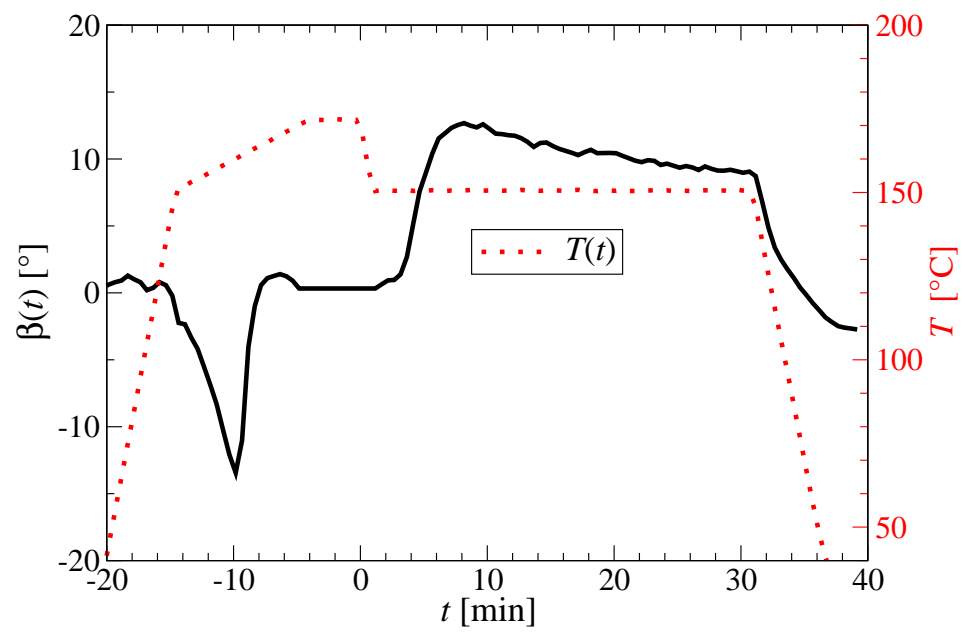

Figure 5. Tilt-angle tracking curve $\beta(t, T)$ from the automatic fiber-mapping procedure in an experiment in which $\beta$ is changing considerably (hard-elastic polypropylene; melt-annealing at $171^{\circ} \mathrm{C}$ and recrystallization at $150^{\circ} \mathrm{C}$ )

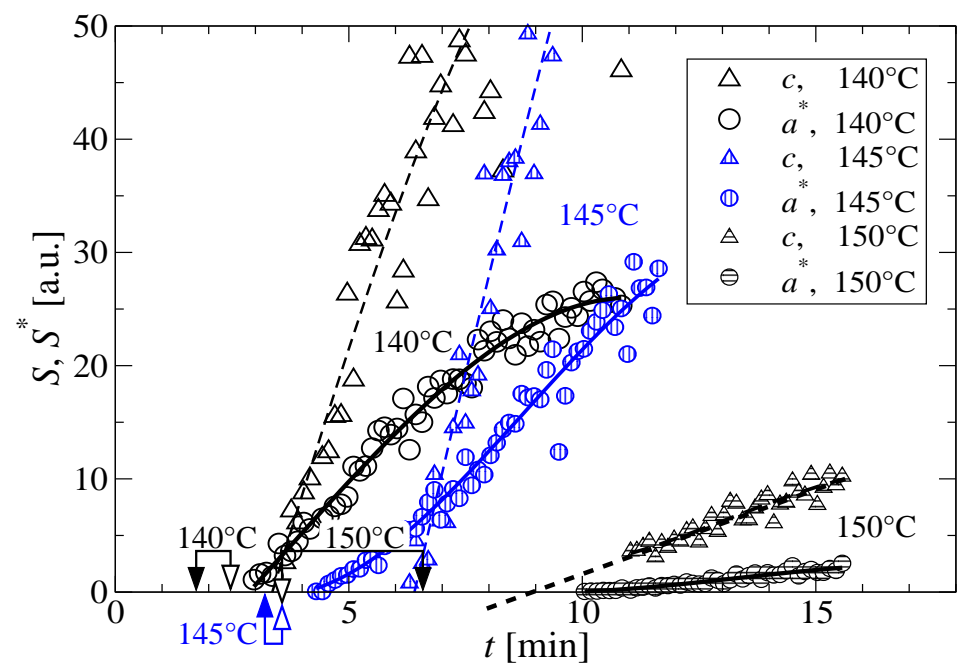

Figure 6. Evolution of relative weight crystallinities $S$, and $S^{*}$ of $c$-oriented crystallites (dashed regression lines) and $a^{*}$-oriented crystals (solid regression lines), resp. during isothermal, oriented crystallization of hard-elastic PP from a quiescent melt as a function of crystallization temperature. Double-head arrows pointing at the $t$-axis indicate the first sighting of the $a^{*}$-set (full arrow head), and of the $c$-set (open arrow head), respectively

\section{Monitoring low-strain mechanical tests with high precision}

Scientific problem. Subjected to mechanical tests, the common thermoplastic polymers fail at much lower strain than elastomers. Thus, the investigation of fatigue and failure mechanisms requires that both the macroscopic mechanical data and nanoscopic structural parameters are monitored with very high accuracy. Searching for advanced polymer composites, new materials must prove their serviceability in mechanical tests. In order to study the underlying mechanisms, the mechanical tests can be monitored by SAXS. With the advent of very high-brilliance synchrotron sources it will soon become possible to record low-noise 2D SAXS patterns with such high cycle frequencies that the mechanical tests can even be performed at strain rates of industrial relevance. Thus, automated methods must be designed 


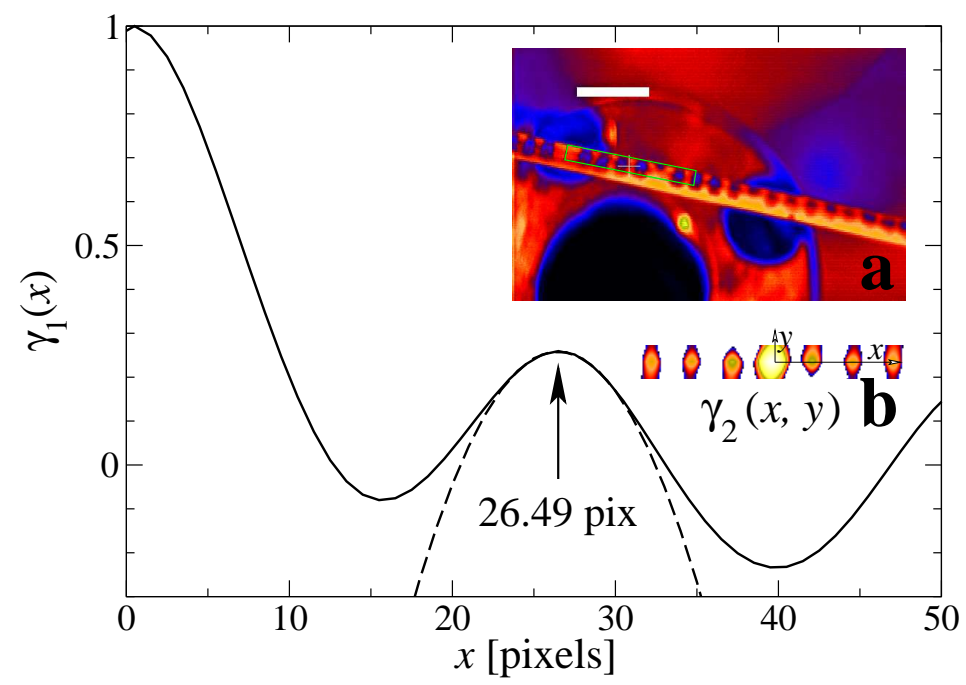

Figure 7. noneStrain from recorded video frames. Inset a: In the first video frame a region of interest (ROI) with fiducial marks is defined. Inset $\mathbf{b}$ : From the ROI the 2D correlation function $\gamma_{2}(x, y)$ is computed. Main drawing: The center of the long-period peak in $\gamma_{1}(x)=\gamma_{2}(x, 0)$ is fitted by a parabola (dashed line) to compute the distance between the fiducial marks

by which the experimental parameters can be extracted with high precision. Our latest methodical development [34] permits to determine the macroscopic strain of the sample at the point of irradiation with an accuracy of 3 decimals. Such high precision is required for thermoplastic materials that fail at low strains $\left(\varepsilon_{b} \approx 0.1\right)$.

Solution. The method evaluates a sequence of video frames taken during the measurement, extracts the grating of the fiducial marks on the sample from the actual video image, computes its 1D correlation function, and evaluates its "long period" peak. The same high-accuracy peak-shape analysis is used to extract nanostructure parameters from SAXS data.

Determination of the macroscopic strain $\varepsilon$ starts from video frames taken of a sample that carries fiducial marks (see Fig. 7a). Once for an experiment the user has to provide some input. It is based on the first image of the series. The center of the X-ray beam on the sample is marked by a cross in the image. Close to this center the user defines a rectangular region of interest (ROI), $\rho_{m}^{\prime}(x, y)$. In Fig. $7 \mathrm{a}$ this region is bordered by a dashed line. $x$ and $y$ are pixel coordinates in the direction of strain and perpendicular to it, respectively. The same ROI is applied to all video frames of the experiment. The ROI itself is structured by the fiducial marks running perpendicular to the straining direction. From such a structure function $\rho_{m}^{\prime}(x, y)$ the 2D correlation function $[35,36]$

$$
\gamma_{2}(x, y)=\frac{\rho_{m}^{\star 2}(x, y)}{\rho_{m}^{\star 2}(0,0)}
$$

is computed, with

$$
\rho_{m}(x, y)=\rho_{m}^{\prime}(x, y)-\bar{\rho}_{m}
$$

representing the fluctuation of $\rho_{m}^{\prime}(x, y)$ about its average $\bar{\rho}_{m}$, and the autocorrelation being defined by the integral

$$
f^{\star 2}(x, y)=\iint_{-\infty}^{\infty} f(u, v) f(u+x, v+y) d u d v .
$$

In Fig. $7 \mathrm{~b}$ the visible caps demonstrate, where $\gamma_{2}(x, y)$ is positive. The macroscopic strain $\varepsilon$ in straining direction is computed from the section $\gamma_{1}(x)=\left\lceil\gamma_{2}(x, y)\right\rceil_{1}(x)$ of $\gamma_{2}$ in straining direction. Figure 7 


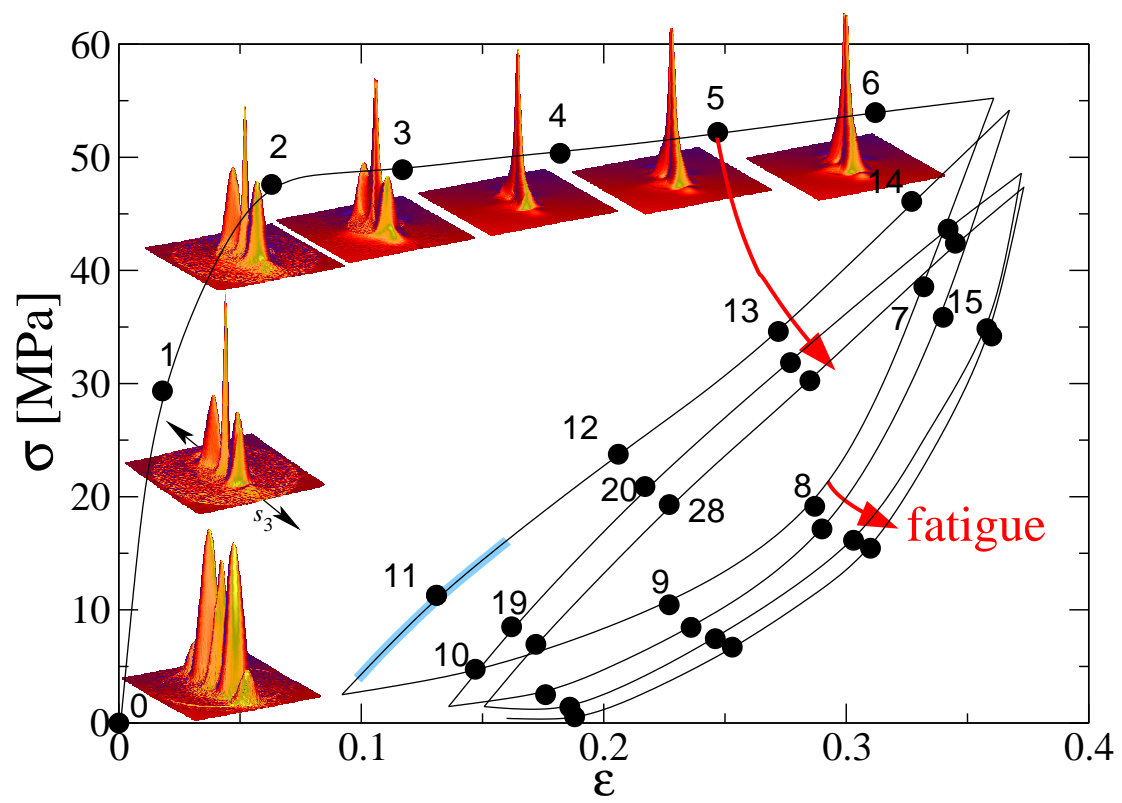

Figure 8. noneOriented PP. Load cycling monitored by SAXS. Circular dots show where SAXS patterns have been recorded. Numerical labels indicate their sequence. The highlighted part of the curve near label 11 indicates the part traversed during the recording of pattern 11. The drawing direction with respect to the patterns, $s_{3}$, is indicated by a double-head arrow

presents this curve and its analysis. Its first peak indicates the long-period. This is the actual average distance between the fiducial marks, $\ell$. A parabola (dashed line) is fitted to the long-period maximum, and the position of its vertex is determined (arrow) with an accuracy of 0.01 pixels. Let $\ell_{0}$ the initial distance between the marks, then the macroscopic strain is $\varepsilon_{m}=\ell / \ell_{0}-1$.

Concerning the scattering patterns, similar analysis is possible. Nevertheless, the peaks observed in the scattering pattern or in the CDF [37] computed from the pattern are no longer 1D but 2D positioned in the plane $\left(s_{12}, s_{3}\right)$ or $\left(r_{12}, r_{3}\right)$, respectively. Again, the user defines a ROI in which the analysis procedure searches for the peak maximum, extracts the peak cap, and fits now a bivariate [38] polynomial of 2 nd order to it. From the coefficients of the polynomial several parameters are readily determined. Most important are the peak position and the widths of the peak in meridional and equatorial directions, respectively. Thus, the evolution of these parameters during the experiment can be tracked automatically.

Application. Slow continuous mechanical tests of oriented PP are monitored [39] by SAXS and quantitatively evaluated by the CDF [37] method. Fatigue is studied in a load-reversal experiment (between 10\% and 35\% strain, Fig. 8). In each cycle crystallization, layer break, and relaxation melting are observed. Figure 9 presents the result. The top chart shows the strain, $\varepsilon(t)$, imposed to the material and determined at the point of X-ray irradiation. The four load-reversal cycles are easily identified. The chart in the middle reports the extracted nanostructure parameters, $L(t), e_{c a c}(t)$, and $S(t)$. The bottom diagram presents the macroscopic stress, $\sigma(t)$, which the material is opposing to strain. The figure shows that crystallization, rupture of lamellae and melting of fragments are continuously reshaping the domains of the PP material in the cycles of the fatigue test. The long-period cycle exhibits a phase shift with respect to the imposed strain cycling. Fatigue is demonstrated in the curve $\sigma(t)$ by decreasing stress peaks. An in-depth discussion of the zones indicated in Fig. 9 is in the original paper [39]. In particular, the combination of SAXS and fatigue test shows the transition from stress-induced crystallization to crystallite rupture $\sigma(t) \approx 20 \mathrm{MPa}$. 


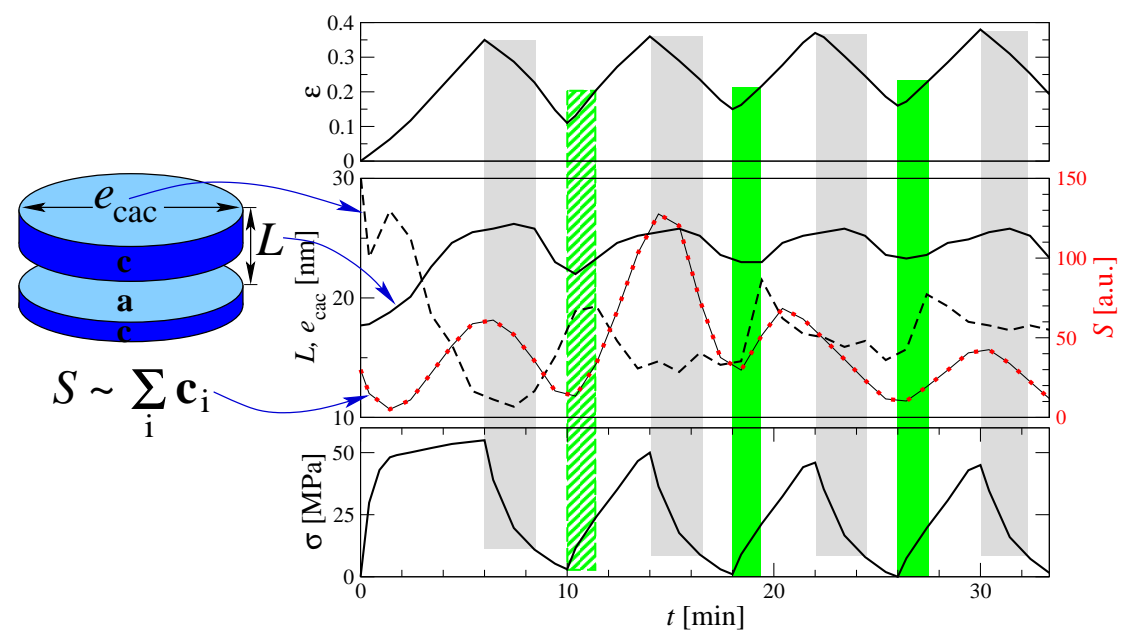

Figure 9. noneDynamic load-reversal mechanical test of hard-elastic PP film. As a function of the elapsed time $t$ the macroscopic parameters strain, $\varepsilon$ (top graph), and tensile stress, $\sigma$ (bottom graph) are displayed together with topological nanostructure parameters (middle). In the middle diagram the solid line shows the long period, $L$. The broken line displays the lateral extension, $e_{c a c}$, of a sandwich made from two crystalline lamellae. The line with circular dots exhibits the variation of the strength, $S$, of the CDF. Vertical bars indicate zones of strain-induced crystallization (dark green) and relaxation-induced melting (light gray), respectively

\section{Conclusions}

During the first decades of synchrotron radiation experiments in polymer science data analysis technique was more advanced than the instruments. This situation has reversed recently. The corresponding data evaluation bottleneck may be remedied by new data evaluation methods that correspond to modern instrumental technique. The presented methods are part of the effort to alleviate the actual problem. Nevertheless, the progress in the field of instrumentation is fast and other measures must be found to keep up. Here it would be possible to move part of the data evaluation business from the individual user to the synchrotron facility. Moreover, standardization and modularization of software could help to further alleviate the problem.

Acknowledgments. The author thanks the Hamburg Synchrotron Radiation Laboratory (HASYLAB) for beam time granted in the frame of project II-04-039. This work has been supported by the 7th framework program of the European Union (Project NANOTOUGH NMP-2007-2.1-1).

[1] Polanyi M 1921 Z. Phys. 7 149-180

[2] Polanyi M and Weissenberg K 1923 Z. Physik 9 123-130

[3] Riekel C and Engström P 1995 Nuclear Instr. Meth. Phys. Res. B97 224-230

[4] Waigh T A, Donald A M, Heidelbach F, Riekel C and Gidley M J 1999 Biopolymers 49 91-105

[5] Kolb R, Wutz C, Stribeck N, v Krosigk G and Riekel C 2001 Polymer 42 5257-5266

[6] Stribeck N, Almendarez Camarillo A, Nöchel U, Schroer C, Kuhlmann M, Roth S V, Gehrke R and Bayer R K 2006 Macromol. Chem. Phys. 207 1239-1249

[7] Stribeck N, Nöchel U, Fakirov S, Feldkamp J, Schroer C, Timmann A and Kuhlmann M 2008 Macromolecules 417637 7647

[8] Bonse U and Busch F 1996 Prog. Biophys. Molec. Biol. 65 133-169

[9] Schroer C G, Kuhlmann M, Roth S V, Gehrke R, Stribeck N, Almendarez Camarillo A and Lengeler B 2006 Appl. Phys. Lett. 88164102

[10] Schroer C G, Kuhlmann M, Günzler T F, Benner B, Kurapova O, Patommel J, Lengeler B, Roth S V, Gehrke R, Snigirev A, Snigireva I, Stribeck N, Almendárez Camarillo A and Beckmann F 2006 Proc. SPIE $63186318 \mathrm{H}$

[11] Kak A C and Slaney M 1999 Principles of Computerized Tomographic Imaging (New York: IEEE Press)

[12] Bracewell R 1999 The Fourier Transform and Its Applications 3rd ed (New York: Mc Graw-Hill) 
[13] Guinier A and Fournet G 1955 Small-Angle Scattering of X-Rays (London: Chapman and Hall)

[14] Hosemann R and Bagchi S N 1962 Direct Analysis of Diffraction by Matter (Amsterdam: North-Holland)

[15] Alexander L E 1979 X-Ray Diffraction Methods in Polymer Science (New York: Wiley)

[16] Glatter O and Kratky O (eds) 1982 Small Angle X-ray Scattering (London: Academic Press)

[17] Feigin L A and Svergun D I 1987 Structure Analysis by Small-Angle X-Ray and Neutron Scattering (New York: Plenum Press)

[18] Baltá Calleja F J and Vonk C G 1989 X-Ray Scattering of Synthetic Polymers (Amsterdam: Elsevier)

[19] Stribeck N 2007 X-Ray Scattering of Soft Matter (Heidelberg, New York: Springer)

[20] Abel N H 1826 J. Reine Angew. Math. 1 153-157

[21] Guinier A and Fournet G 1947 Nature 160501

[22] DuMond J W M 1947 Phys. Rev. 72 83-84

[23] Bitter I, Kaufman A E and Sato M 2001 IEEE Trans. Visualization and Computer Graphics 7 195-206

[24] Dasch C J 1992 Applied Optics, 31 1146-1153

[25] Dribinski V, Ossadtchi A, Mandelshtam V A and Reisler H 2002 Rev. Sci. Instr. 73 2634-2642

[26] Fraser R D, Macrae T P, Miller A and Rowlands R J 1976 J. Appl. Cryst. 9 81-94

[27] Stribeck N 2009 Acta Cryst. A65 46-47

[28] Franklin R E and Gosling R G 1953 Acta Cryst. 6 678-685

[29] Stribeck N and Nöchel U 2009 J. Appl. Cryst. 42 295-301

[30] Stribeck N 2008 Downloads http://www.chemie.uni-hamburg.de/tmc/stribeck/dl

[31] Stribeck N, Nöchel U and Funari S S 2009 Macromolecules 42 2093-2101

[32] Mencik Z 1972 J. Macromol. Sci. Phys. B6 101-115

[33] VNI 2007 Pv-wave manuals V 7.5, Houston, TX, USA

[34] Denchev Z, Dencheva N, Funari S S, Motovilin M, Schubert T and Stribeck N 2009 J. Polym. Sci. Part B: Polym. Phys. in print

[35] Vonk C G and Kortleve G 1967 Colloid Polym. Sci. 220 19-24

[36] Strobl G R and Schneider M 1980 J. Polym. Sci., Part B: Polym. Phys. B18 1343-1359

[37] Stribeck N 2001 J. Appl. Cryst. 34 496-503

[38] Hall E L 1980 Computer Image Processing and Recognition (London: Academic Press)

[39] Stribeck N, Nöchel U, Funari S S, Schubert T and Timmann A 2008 Macromol. Chem. Phys. 209 1992-2002 